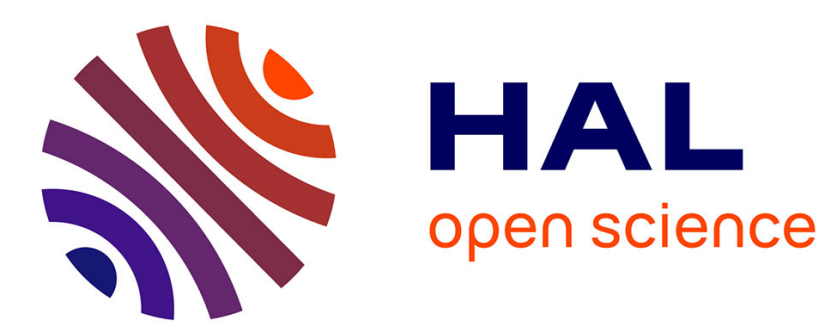

\title{
Variations of tomography signals in shallow water due to bottom topography irregularities
}

\author{
M. Taroudakis
}

\section{To cite this version:}

M. Taroudakis. Variations of tomography signals in shallow water due to bottom topography irregularities. Journal de Physique IV Proceedings, 1994, 04 (C5), pp.C7-1079-C7-1082. 10.1051/jp4:19945237 . jpa-00252924

\section{HAL Id: jpa-00252924 https://hal.science/jpa-00252924}

Submitted on 1 Jan 1994

HAL is a multi-disciplinary open access archive for the deposit and dissemination of scientific research documents, whether they are published or not. The documents may come from teaching and research institutions in France or abroad, or from public or private research centers.
L'archive ouverte pluridisciplinaire HAL, est destinée au dépôt et à la diffusion de documents scientifiques de niveau recherche, publiés ou non, émanant des établissements d'enseignement et de recherche français ou étrangers, des laboratoires publics ou privés. 


\title{
Variations of tomography signals in shallow water due to bottom topography irregularities
}

\section{M.I. TAROUDAKIS}

Foundation for Research and Technology-Hellas, Institute of Applied and Computational Mathematics, P.O. Box 1527, 71110 Heraklion Crete, Greece and University of Crete, Department of Mathematics, P.O. Box 1470, 71409 Heraklion Crete, Greece

\begin{abstract}
The paper presents a quantitative study of the effect of the bottom topography on the acoustic signals used in ocean acoustic tomography applications. The study is restricted to shallow water areas where the importance of the bottom boundary in modelling acoustic propagation is more pronounced. It is shown that significant variations of the signal are expected due to the presence of some local boundary irregularity, being of the same order as regards the "useful" variations used for the data inversions. It is concluded that an accurate bottom description is needed to be used in connection with an reliable model handling acoustic propagation in range-dependent environments for all ocean acoustic tomography applications.
\end{abstract}

\section{INTRODUCTION}

Ocean acoustic tomography is presently applied mainly in deep water areas for monitoring oceanographic processes in the sea environment. At the same time shallow water tomography is a subject that has drawn the interest of many scientists, and modelling problems for shallow water tomography are currently under research. Ray-theoretic tomography schemes seem to be inappropriate at these areas, and therefore most efforts in this field are made using wave-theoretic considerations, mainly matched field techniques and modal tomography $[1,2,3]$. The appropriate measurements are either the acoustic field in the time domain at a specific point due to a pulsed source, or the field at a vertical (or horizontal) array of hydrophones due to a $\mathrm{cw}$ source, from which its modal content is extracted.

Since the problem deals with acoustic propagation in shallow water, where the interaction with the sea bottom is very important, the accurate knowledge of the bottom nature and the topography variations is necessary. Most of the known works on shallow water tomography neglect the possibility of sound propagation over an irregularly shaped bottom boundary.

The present work deals with the problem of the assessment of bottom topography influence on the useful tomographic measurements in both the frequency and the time domain. Focusing our work to modal acoustic tomography, the quantities influenced by bottom topography irregularities and related to the inversion schemes are the modal phase and the modal arrival times of the acoustic signal. Their variations when a sea-mount is present in the water-bottom interface are calculated using both adiabatic-mode and coupledmode theory. First approach is very weak in cases of strong environmental variations (either in the water column or in the bottom boundary), while coupled-mode theory gives the most accurate results for strongly range-dependent environments.

\section{OCEAN ACOUSTIC TOMOGRAPHY BASED ON NORMAL-MODE THEORY}

The acoustic equation governing sound propagation in the ocean is usually written in the frequency domain (Helmholtz equation). For an axially symmetric environment the homogeneous equation in cylindrical co-ordinates is

$$
\nabla^{2} p(r, z)+\frac{1}{\rho(z)} \nabla \rho(z) \cdot \nabla p(r, z)+k^{2}(r, z) p(r, z)=0,
$$

where, $p(r, z)$ is the sound pressure, $\rho(z)$ is the density which is assumed to be depth-dependent only, and $k(r, z)=\omega / c(r, z)$ is the wavenumber. The equation is supplemented by appropriate boundary and radiation conditions to form an elliptic problem with unique solution. 
Ocean acoustic tomography deals with the problem of determining the sound speed structure, that is the determination of the function $c(r, z)$ by means of acoustic measurements. The most common approach is to assume a known background environment with sound speed structure described by $c_{0}(r, z)$ and then try to calculate sound speed differences $\delta c(r, z)$ so that the sound speed structure of the actual environment is given by

\subsection{Modal phase approach}

$$
c(r, z)=c_{0}(r, z)+\delta c(r, z)
$$

Using normal-mode theory, the sound speed variations are associated with measurable modal quantities of the acoustic field. Using a $\mathrm{cw}$ source and mode filtering at an array of hydrophones moored at range $R$ the adiabatic modal phase

$$
\Phi_{n}(R)=\int_{0}^{R} k_{n}(r) d r
$$

can me determined, where $k_{n}(r)$ are the eigenvalues of the depth equation determined at each range r. Association of this quantity with that of the background environment together with an approximate relationship between the eigenvalues of the actual and the reference field [4] lead to the expression

$$
\delta \Phi_{n}(R)=-\int_{0}^{R} \frac{1}{k_{n}^{0}(r)} \int_{0}^{\infty} \rho_{0}^{-1}(r)\left|u_{n}^{0}(z ; r)\right|^{2} k_{0}^{2}(r, z) \frac{\delta c(r, z)}{c_{0}(r, z)} d z d r
$$

relating the unknown quantity $\delta c(r, z)$ with the measured modal phase differences and known quantities of the reference environment. This formula is the basis of modal phase inversion schemes [1, 3]. The modal phase difference being the input data of the problem should be calculated with great accuracy so as the reliability of the inverse scheme is ensured.

\subsection{Modal travel time approach}

Consider now a pulsed source described by the source excitation function $X_{C}(\omega)$ Using concepts of linear filter theory, the signal $\hat{X}_{D}(\omega)$ at the receiver is given by

$$
\hat{X}_{D}(\omega)=H_{C D}(\omega) X_{C}(\omega)
$$

where $H_{C D}(\omega)$ is the transfer function of the environment representing the sound pressure for a cw source of frequency $\omega$. Using inverse Fourier transform, the signal in the time domain at the receivers' location is calculated:

$$
\hat{X}_{D}(t)=\mathcal{F}^{-1}\left[\hat{X}_{D}(\omega) ; \omega \rightarrow t\right]
$$

The peaks of the signal arrival pattern in many cases represent modal arrivals, that is energy propagating at specific modes. According to the theory, normal modes at a narrow band of a central frequency $\omega_{0}$ propagate at a group velocity.

$$
v_{g, n}=\left.\frac{\partial \omega}{\partial k_{n}}\right|_{\omega_{0}}
$$

The associated modal arrival times for $k_{n}$ varying in range are therefore:

$$
t_{n}(R)=\left.\int_{0}^{R} \frac{\partial k_{n}(r) d r}{\partial \omega}\right|_{\omega_{0}}=\left.\frac{\partial \Phi_{n}(R)}{\partial \omega}\right|_{\omega_{0}}
$$

Considering a background (reference) environment and an actual one whose velocity structure is to be recovered, the corresponding travel time differences of the modal peaks of the arrival pattern are given by formula

$$
\delta t_{n}(R)=\left.\frac{\partial \delta \Phi_{n}(R)}{\partial \omega}\right|_{\omega_{0}}
$$

This formula can be used in connection with equation (3) to determine an inverse problem which is solved for the unknowns $\delta c$.

Since the time differences between the various peaks are calculated by comparing the arrival pattern of the tomographic signal for the reference environment with that of the actual one which is measured at a single receiver placed at range $R$, the accurate calculation of the system transfer function of the reference environment is essential for the accuracy of the inversion results. This is particularly true in the case of a range-dependent environment such as the one with a bottom irregularity,

When a bottom topography of irregular character exists in the water area, an adiabatic normal mode approach would lead to erroneous results especially when the bottom irregularity is accompanied by a water 
irregularity such as a sea eddy, which is the desired oceanographic structure to be recovered. In the next section a coupled-mode formula for the calculation of the system transfer function is proposed. Using this approach the system transfer function is accurately calculated for any case of range-dependency and therefore the calculated travel time differences are accurate.

\section{A COUPLED-MODE SOLUTION FOR THE TRANSFER FUNCTION}

When the sea environment is characterised by parameter variations of compact support, a suitable discretization of the environment in vertical rings (segments) of constant topography and range-independent parameters and the application of a variational principle lead to the following coupled-mode solution of the acoustic field

$$
p^{i}(r, z)=\sum_{n}\left[A_{n}^{i} H_{0}^{(1)}\left(k_{n}^{i} r\right)+B_{n}^{i} H_{0}^{(2)}\left(k_{n}^{i} r\right)\right] u_{n}^{i}=H_{C D}(\omega)
$$

where the index $i$ denotes the segment at which the receiver is placed. The coefficients $A_{n}^{i}$ and $B_{n}^{i}$ are calculated solving a linear system of equations determined by the application of the continuity conditions at the artificial vertical boundaries of the environment [5]. $H_{0}^{(.)}$are Hankel functions of 1st and 2nd order.

Using this formula for the calculation of the system transfer function one could obtain accurate predictions of the signal arrival pattern of a pulsed source (Equations (4) and (5)).

\section{NUMERICAL RESULTS}

Consider the model environment presented in Figure 1. The eddy existing in the water column can be viewed as the structure to be recovered by ocean acoustic tomography. We will focus our efforts to modal travel time tomography and we will assume that the environment is the reference one for which we will calculate the signal arrival pattern for an environment with a sea-mount of $15 \mathrm{~m}$ bottom elevation and another one with a flat bottom using the coupled-mode approach. The calculated modal travel time differences between the two patterns is a measure of the bottom influence on the tomographic signal. In both cases a known bottom consisting of fluid materials $\left(\rho=1500 \mathrm{~kg} / \mathrm{m}^{3}\right.$ and $c=1600 \mathrm{~m} / \mathrm{sec}$ ) will be assumed. The frequency of the $\mathrm{cw}$ source which is placed at $60 \mathrm{~m}$ depth is taken to be $110 \mathrm{~Hz}$. The pulsed source has the same central frequency, $20 \mathrm{~Hz}$ bandwidth and gaussian shape. For comparison reasons adiabatic modal travel time estimations are also performed. The receiver is placed at $60 \mathrm{~m}$ depth and $50000 \mathrm{~m}$ range.

Figure 2 presents the arrival patterns of the tomographic signal with and without the sea-mount. The corresponding travel time differences for the 9 propagating modes appear in table I, second column. Third column presents the adiabatic travel time differences, while the fourth column presents the modal travel time variations for the case of the flat bottom due to the presence of an "actual" eddy which is to be recovered. Recall that the values of this column are used for acoustic tomography when bottom topography irregularities are neglected. Finally, the adiabatic modal phase differences of the acoustic field of a $110 \mathrm{~Hz}$ $\mathrm{cw}$ source for the two cases of the reference environment are presented in column 5 , while column 6 presents modal phase differences between the flat reference environment and the actual one. This difference is used in modal phase ocean acoustic tomography when the bottom boundary irregularities are neglected

Table I

Signal variations due to the bottom irregularity

R: Reference, M: 15m sea-mount, (C): Coupled, (A): Adiabatic, A: Actual

\begin{tabular}{|r|cc|c||lc|}
\hline Mode & \multicolumn{2}{|c||}{ Arrival time differences (msec) } & \multicolumn{2}{c|}{ Modal phase differences (rad) } \\
\cline { 2 - 6 } Order & R-M(C) & R-M(A) & R-A(C) & R-M(A) & R-A(A) \\
\hline 1 & - & -0.47 & - & -0.245 & -10.875 \\
2 & -7.33 & -1.95 & 14.65 & -1.046 & -10.879 \\
3 & - & -4.71 & & -2.694 & -10.305 \\
4 & -80.57 & -8.68 & 14.65 & -5.241 & -9.633 \\
5 & -25.63 & -13.49 & 15.87 & -8.796 & -9.283 \\
6 & 34.18 & -19.05 & 25.63 & -13.134 & -9.225 \\
7 & -93.50 & -25.12 & 12.20 & -18.395 & -9.419 \\
8 & -24.42 & -31.04 & 15.87 & -24.121 & -9.629 \\
9 & -170.9 & -31.57 & 13.43 & -30.844 & -9.581 \\
\hline
\end{tabular}

By comparing the contents of the second and the fourth column, it can be seen that the signal variations due to the presence of the sea-mount are comparable with them of the "actual" field with respect to the reference one. Similar results can be obtained for different environments being clearly an indication of the 


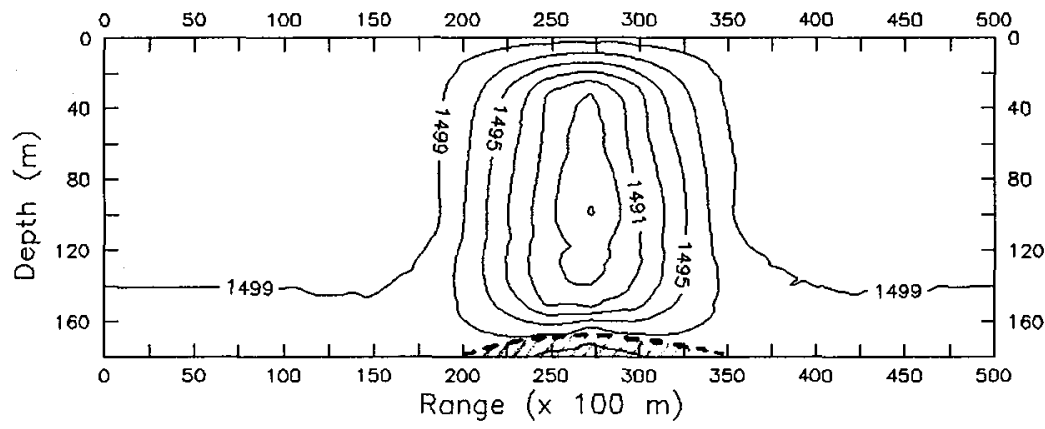

Figure 1: The reference environment (with and without the sea-mount)

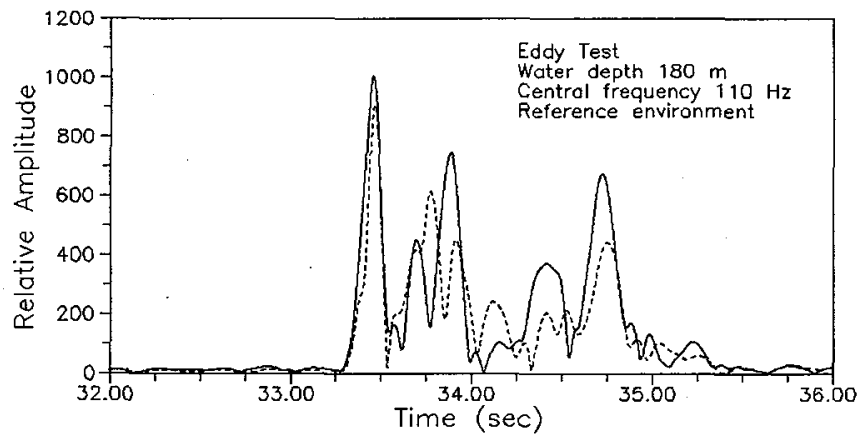

Figure 2: Arrival patterns of a gaussian pulse at the reference environment with (dotted line) and without the sea-mount (solid line)

importance of the bottom bathymetry for shallow water ocean acoustic tomography. At the same time the comparison of the fifth and the sixth column leads to the same result as regards the modal phase tomography.

\section{CONCLUSIONS}

The sea bottom topography has shown to be of significant importance on the modal quantities used as input data for ocean acoustic tomography. This was well illustrated in the presented example even though the sea mount had a small height. Since the accurate calculation of the system transfer function is essential in all applications of ocean acoustic tomography, coupled-mode schemes seem to be appropriate tools for obtaining the acoustic field especially in shallow water environments

\section{Acknowledgements}

I would like to express my thanks to Ms Giwta Tzompanopoulou for her computational assistance. This work was partly funded by EC under contract MAST-0008-C(MB) THETIS.

\section{References}

[1] Shang E.C., "Ocean Acoustic Tomography Based on Adiabatic Mode Theory" J.Acoust.Soc.Am., 85 (1989), 1531-1537.

[2] Tolstoy A. Diachok O. and Frazer L.N. "Acoustic tomography via matched field processing," J.Acoust.Soc.Am. 89 (1991), 1119-1127.

[3] Taroudakis M.I. and Papadakis J.S. "Reconstruction of sound velocity profiles in the Sea Using Ocean Acoustic Tomography Techniques" in European Conference on Underwater Acoustics edited by M.Weydert (Elsevier 1993) pp 607-610.

[4] Rajan S., Lynch J.F. and Frisk G.V. "Perturbative Inversion Methods for Obtaining Bottom Geoacoustic Parameters in Shallow Water" J.Acoust.Soc.Am., 82 (1987), 998-1017.

[5] Taroudakis M.I., Athanassoulis G.A and Ioannidis J.P. "A hybrid solution of the Helmholtz equation in shallow water based on a variational principle" in Acoustique Sous-Marine et Ultrasons, (LMA, Marseille 1991) pp 213-227. 